\title{
A Simplified Method for Predicting Tunneling-Induced Ground Movement considering Nonuniform Deformation Boundary
}

\author{
Xuepeng Zhang $\mathbb{D}^{1,2,3}$ Yujing Jiang, ${ }^{1,4}$ Yue Cai $\mathbb{D}^{5},{ }^{5}$ Xin $\mathrm{Li}^{6}{ }^{6}$ Naser Golsanami $\mathbb{D}^{1}$, \\ Xiao Wang, ${ }^{7}$ Jian Hao, ${ }^{1}$ Ningbo Li, ${ }^{4}$ Fabo Wu, ${ }^{8}$ and Xiaohan Wang ${ }^{1}$ \\ ${ }^{1}$ State Key Laboratory of Mining Disaster Prevention and Control Co-Founded by Shandong Province and \\ the Ministry of Science and Technology, Shandong University of Science and Technology, Qingdao 266590, China \\ ${ }^{2}$ Key Laboratory of Deep Earth Science and Engineering (Sichuan University), Ministry of Education, Chengdu 610065, China \\ ${ }^{3}$ Key Laboratory of Rock Mechanics and Geohazards of Zhejiang Province, Shaoxing University, Shaoxing 312000, China \\ ${ }^{4}$ Department of Civil Engineering, Nagasaki University, Nagasaki 852-8521, Japan \\ ${ }^{5}$ Fukumichi Limited Liability Company, Fukuoka 813-0041, Japan \\ ${ }^{6}$ China Xiong'an Group, Xiong'an 071700, China \\ ${ }^{7}$ School of Civil Engineering, Southeast University, Nanjing 211189, China \\ ${ }^{8}$ College of Civil Engineering and Architecture, Shandong University of Science and Technology, Qingdao 266590, China
}

Correspondence should be addressed to Yue Cai; ycai2020@naturelife.minibird.jp and Naser Golsanami; golsanami_naser@ yahoo.com

Received 11 July 2021; Revised 30 November 2021; Accepted 9 December 2021; Published 13 January 2022

Academic Editor: Shan Gao

Copyright (c) 2022 Xuepeng Zhang et al. This is an open access article distributed under the Creative Commons Attribution License, which permits unrestricted use, distribution, and reproduction in any medium, provided the original work is properly cited.

\begin{abstract}
Stochastic medium (SM) theory is a practical method in ground settlement prediction, while its nonintegrable double integral form makes the solution process complicated. A simplified analytical solution based on the SM theory is developed to predict the ground movement in tunneling excavation. With the simplified solution, the ground movement for single tunnel and twin tunnels could be predicted based on the gap parameter $G$ and influence angle $\beta$. A feasible approach is developed to estimate these two parameters using the maximum ground settlement $S_{\max }$ and tunnel design parameters, including tunnel depth $H$ and diameter $R$. The proposed approach can be used to predict the ground movement curve for both circular and noncircular cross section tunnels. To validate its accuracy, the results predicted by the simplified procedure are compared with those obtained by the SM theory and measured in situ. The comparisons show that the current results agree well with those obtained by the SM theory and measured in situ. The comparison of five tunnels in literature illustrates that the simplified method can provide a more reasonable prediction for the ground movement induced by tunneling.
\end{abstract}

\section{Introduction}

In current urban areas, shallow-buried tunnel such as the subway has become much more popular as it can provide the largest traffic volume and the fastest transportation speed to solve the traffic congestion [1]. Ground movement induced by tunneling is inevitable $[2,3]$. Tunneling-induced ground movement could damage surrounding buildings or infrastructures and result in serious environmental problems. It is, therefore, necessary to predict tunneling-induced ground movement by engineers. Much research has been carried out regarding this issue throughout empirical equation method $[4,5]$, analytical solution method $[6,7]$, numerical simulation method $[8,9]$, and model test method [10]. Besides, the machine learning method provides new solutions for tunneling-induced ground settlement, including the artificial neural network [11-13], support vector machine [14-16], and random forest $[17,18]$.

Among these methods, the analytical solution method is widely used in practice to predict the ground surface 
settlement. Some attempts, which are subjected to limitations, have been made to obtain a closed solution of ground surface settlement. For instance, Verruijt and Booker and Loganathan and Poulos assumed that ground materials behave linearly elastic to derive the ground movement formulas $[6,19]$. In practice, the strength envelopes of geomaterials are nonlinear, and the linear relationship is just a special case [20-23]. Consequently, the predicted ground movement differs from the in situ or experimental observed values. As the overlying strata behave in a complex manner, and the ground movement is governed by a series of known and unknown factors, such as tunnel construction method, tunnel-driving details, tunnel depth, and diameter, initial stress state, and stress-strain behavior of the soil around tunnel, the stochastic medium (SM) theory was proposed to predict the tunneling-induced ground movement $[6,24]$. Furthermore, Liu et al. developed the SM theory to estimate the ground surface movement and deformation caused by tunneling [25]. Meanwhile, for the more actual situation, the nonuniform convergence of the tunnel deformation model was introduced to establish ground settlement prediction model [26-29].

For SM theory, because its formula is a nonintegrable double integral, the solution process is rather complicated. Therefore, the SM theory is simplified to predict the ground surface movement [7]. However, how to determine the uniform convergence value of the tunnel cross section as input parameters is not stated clearly, which would affect the accuracy of the predicted results. A more accurate result was achieved using the gap parameter $G$ considering the nonuniform convergence and the main influence angle $\beta$. According to the suggestion by Lee et al., the gap parameter $G$ consists of three parts: the physical gap, the equivalent three-dimensional (3D) elastoplastic deformation at the tunnel face, and workmanship factors [30]. The calculation method for the gap parameter $G$ suggested by Lee requires a few parameters, and the process is relatively complicated. When the in situ information is not enough, the parameter $G$ could be obtained by another simplified approach. The main influence angle $\beta$ is another primary factor that influences the settlement profile of the ground surface. Although the range of influence zone is related to many factors, the geological environment, the physical properties of overlying strata, and the depth of tunnel should be concerned mainly.

Therefore, a simplified analytical solution in the current study is introduced to predict the ground movement in tunneling excavation based on the SM theory. With the simplified solution, the ground movement for single tunnel and twin tunnels could be predicted based on the gap parameter $G$ and influence angle. A feasible approach is developed to estimate these two parameters using the maximum ground settlement $S_{\max }$ and tunnel design parameters, including tunnel depth $H$ and diameter $R$. To validate its accuracy, the predicted results using the simplified procedure are compared with the SM theory and in situ measured values.

\section{Basic Stochastic Model for Tunnel Excavation}

A stochastic model is a tool to estimate probability distributions of potential outcomes by allowing random variation in one or more inputs over time. To calculate the movement of a surface point using the stochastic model, an extraction block can be divided into infinitesimal extraction elements. According to the principle of the combined subsidence effect of the blocks under extraction, the consequence for the extraction block would be equal to the sum of the effects caused by those infinitesimal extraction elements. For an element system, the stratum corresponds to the global coordinate $(x, y, z)$, while the excavation adopts the local coordinate $(\xi, \zeta, \eta)$, as shown in Figure 1. The elemental excavation dimension is defined as $\mathrm{d} \xi, \mathrm{d} \zeta$, and $\mathrm{d} \eta$. The tunnel excavation problem could be simplified as a plane-strain problem. By assuming that each element of excavation collapse completely in the whole excavation zone $\Omega$, the ground surface settlement is obtained by applying the superposition principle, which is [31]

$$
W(x)=\iint_{\Omega} \frac{\tan \beta}{\eta} \exp \left[-\frac{\pi \tan ^{2} \beta}{\eta^{2}}(x-\xi)^{2}\right] \mathrm{d} \xi \mathrm{d} \eta,
$$

where $W(x)$ is the ground surface settlement and $\beta$ is the influence angle of ground settlement.

Generally, the displacement at tunnel wall would be converged by itself or by artificial support. Suppose the initial tunnel section is $\Omega$ and the radial region of the convergence section is $\omega$, as shown in Figure 1. The ground movement is induced by the difference, $\Omega-\omega$, between the initial excavation and the convergence section. Hence, the ground surface settlement $W(x)$ and the horizontal displacement of ground surface $U(x)$ are expressed as follows [31]:

$$
\begin{aligned}
& W(x)=W_{\Omega}(x)-W_{\omega}(x)=\iint_{\Omega-\omega} \frac{\tan \beta}{\eta} \exp \left[-\frac{\pi \tan ^{2} \beta}{\eta^{2}}(x-\xi)^{2}\right] \mathrm{d} \xi \mathrm{d} \eta, \\
& U(x)=U_{\Omega}(x)-U_{\omega}(x)=\iint_{\Omega-\omega} \frac{(x-\xi) \tan \beta}{\eta} \exp \left[-\frac{\pi \tan ^{2} \beta}{\eta^{2}}(x-\xi)^{2}\right] \mathrm{d} \xi \mathrm{d} \eta .
\end{aligned}
$$




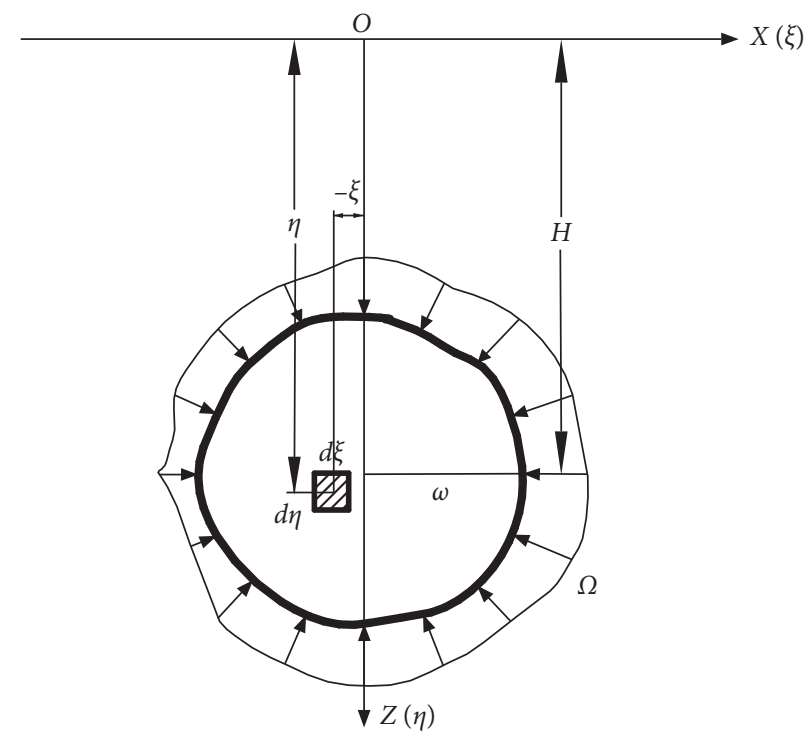

Figure 1: Ground loss of single tunnel excavation [7].

To obtain the magnitude of the ground surface settlement, the parameter values of $\beta$ and the difference of tunnel section induced by convergence should be determined first. Previous research indicated that the difference of the calculated results between the uniform mode and the nonuniform convergence mode would become larger with a much shallower tunnel, resulting from the deformation of the tunnel lining itself with a shallower buried depth [26]. Therefore, the value of $\Omega-\omega$ is very important for calculating surface settlement in shallow tunnels and can affect the accuracy of the predicted values.

\section{Nonuniform Radial Displacement Boundary Condition of Shallow Tunnel}

The excavation section of the tunnel has various shapes in construction. It is often simplified as a circular one for convenience. Considering the nonhydrostatic pressure of the ground, the shape of the tunnel section would become an ellipse after convergence. Therefore, an initial elliptical tunnel section is adopted for discussion in this study. The circular tunnel section would be a special case here. It is assumed that the radial displacement at the tunnel wall is nonuniform, and the tunnel section would still be an ellipse even after convergence.

S-3 mode is suitable for predicting ground surface subsidence and horizontal deformation [32]. The displacement of tunneling openings could be estimated by following the fundamental modes, as shown in Figure 2. They assumed the deformation of the excavation surface mainly consists of (1) uniform radial displacement or deformation $u_{0}$ induced by ground loss, (2) ovalization deformation $u_{\theta}$ of the tunnel section without ground loss, such as the upheaved deformation at the tunnel bottom induced by stress release and a lateral elongation of the circular section, and (3) rigid vertical settlement $u_{z}$ of the whole tunnel because of gravity.

Figure 3 illustrates the calculation model for single and twin tunnels before and after convergence. For simplification, the tunnel section in the twin-tunnel model is assumed to be an ellipse, and the geometry size of the twin tunnels is the same. The major axis of the ellipse is defined as A, and the minor axis is defined as B correspondingly. In case of a circle tunnel section, the major axis of the ellipse equals the minor axis. The ground cover of the initial tunnel section is assumed to be $H$. For twin tunnels, the distance between the two tunnel centers is $L$. Considering the rigid vertical settlement of the tunnel $u_{z}$, the location of the tunnel center after convergence would be $H+u_{z}$. The major axis of the ellipse becomes $A-u_{o}+u_{\theta}$, and the minor axis becomes $B-u_{o^{-}}$ $u_{\theta}$. In case of the S-3 convergence mode, the rigid vertical settlement uz will be $u_{o} / 2$, and the ovalization deformation of tunnel section because of stress release $u_{\theta}$ will also be $u_{o} / 2$ correspondingly.

The ground surface settlement $W(x)$ and the horizontal displacement of ground surface $U(x)$ could be rewritten as follows:

$$
\begin{aligned}
& W(x)=\int_{a}^{b} \int_{c}^{d} \frac{\tan \beta}{\eta} \exp \left[-\frac{\pi \tan ^{2} \beta}{\eta^{2}}(x-\xi)^{2}\right] d \xi d \eta-\int_{e}^{f} \int_{g}^{h} \frac{\tan \beta}{\eta} \exp \left[-\frac{\pi \tan ^{2} \beta}{\eta^{2}}(x-\xi)^{2}\right] \mathrm{d} \xi \mathrm{d} \eta, \\
& U(x)=\int_{a}^{b} \int_{c}^{d} \frac{(x-\xi) \tan \beta}{\eta^{2}} \exp \left[-\frac{\pi \tan ^{2} \beta}{\eta^{2}}(x-\xi)^{2}\right] d \xi d \eta-\int_{e}^{f} \int_{g}^{h} \frac{(x-\xi) \tan \beta}{\eta^{2}} \exp \left[-\frac{\pi \tan ^{2} \beta}{\eta^{2}}(x-\xi)^{2}\right] \mathrm{d} \xi \mathrm{d} \eta,
\end{aligned}
$$




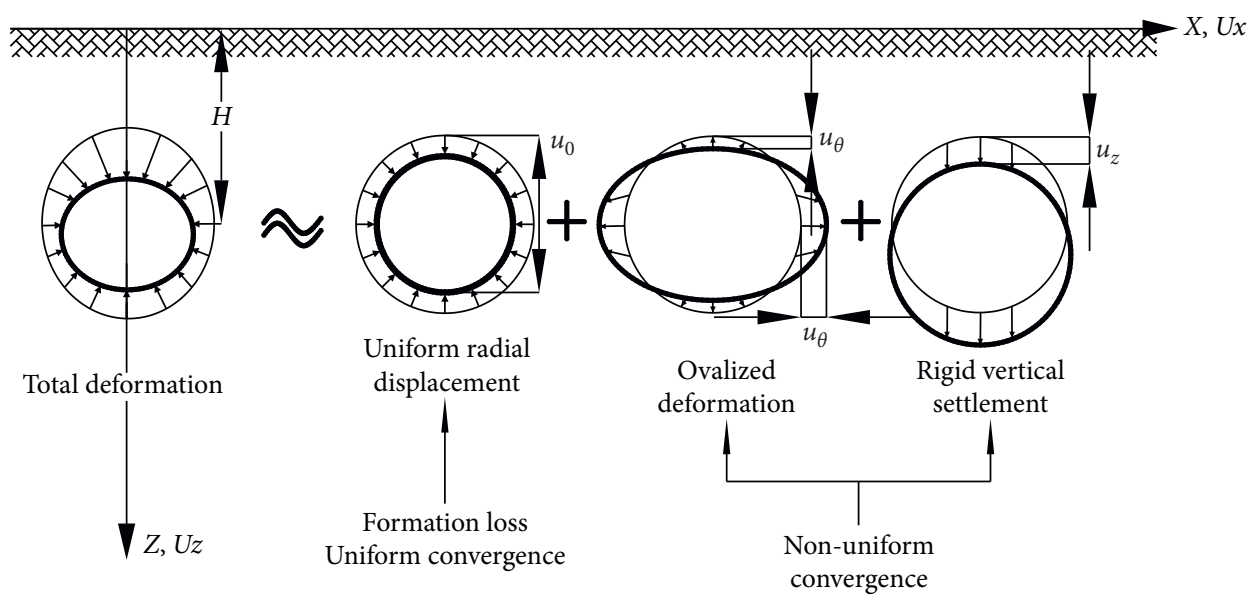

FIGURE 2: Sketch of asymmetrical convergence deformation mode $\left(u_{0}\right.$ denotes the uniform radial displacement; $u_{\theta}$ denotes the ovalized deformation, and $u_{z}$ denotes the rigid vertical settlement).

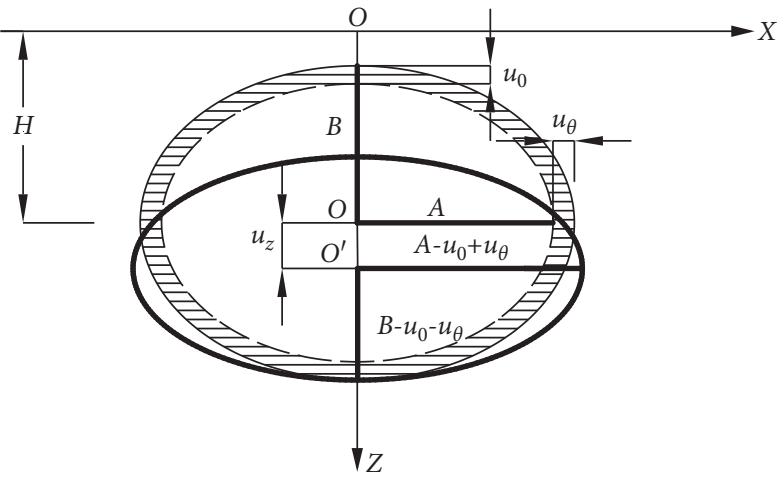

(a)

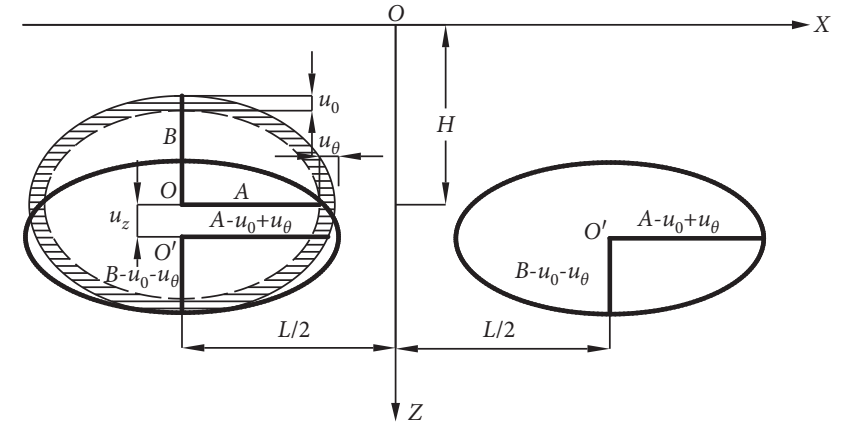

(b)

Figure 3: Calculation model for single and twin tunnels before and after convergence. (a) Model for single tunnel. (b) Model for twin tunnels (modified after Yang's solution [7]).

where $a, b, c$, and $d$ are the boundary parameters of the initial cross section $\Omega$ and $e, f, g$, and $h$ are the boundary parameters of the tunnel cross section $\omega$ after convergence. To obtain the solution of equation (7), the boundary conditions along axis $Z$ and axis $X$ have to be divided into subsections. Those boundary parameters considering the nonuniform boundary condition for single and twin tunnels are presented in Appendices A and B.

Determination of the parameters $u_{o}$ and $\beta$ is required to obtain the solution to this problem. According to the former studies, the radial displacement $u_{o}$ could be estimated by a gap parameter $G$ [33]. The gap parameter is influenced by multiple factors such as the diameter of the tunneling machine, ground condition, and workmanship. The parameter $\beta$ is the angle of influence zone of ground settlement. The details about the gap parameter $G$ and angle $\beta$ will be discussed later. Once the parameters are determined, the problem could be solved by combining equation (3) and the boundary condition.

\section{Simplified Solution of Ground Movement Prediction}

4.1. Simplified Solution for Single Tunnel. The tunnel excavation problem can be simplified as a plane-strain problem. The element excavation is infinite on the $y$-axis, which is along the direction of the tunnel. The final ground surface settlement $W_{e}(x)$ caused by element excavation in an isotropic medium is [31]

$$
W_{e}(x)=\frac{\tan \beta}{\eta} \exp \left[-\frac{\pi \tan ^{2} \beta}{\eta^{2}}(x-\xi)^{2}\right] \mathrm{d} \xi \mathrm{d} \eta,
$$

where $\mathrm{d} \xi \mathrm{d} \eta$ is the area of an element. According to equation (4), the ground surface settlement caused by element excavation is obtained for the plain-strain condition. Converting the $\mathrm{d} \xi \mathrm{d} \eta$ in equation (5) into the area of initial excavation face, the ground surface settlement, due to the complete collapse of the tunnel section $\Omega$, is expressed as [7] 


$$
W_{\Omega}(x)=S_{\Omega} \frac{\tan \beta}{H} \exp \left[-\frac{\pi \tan ^{2} \beta}{H^{2}} x^{2}\right],
$$

where $S_{\Omega}$ is the area of the initial tunnel section and can be defined as $S_{\Omega}=\pi R^{2}$ in case of a circular initial tunnel section and $R$ is the radius of a circular tunnel section. In case of an ellipse section, the radius could be expressed as $R=(A+B) / 2$.

Similarly, for the nonuniform convergence displacement in S-3 mode, the ground surface settlement due to the complete collapse of the tunnel cross section $\omega$ could be written as

$$
W_{\omega}(x)=S_{\omega} \frac{\tan \beta}{H} \exp \left[-\frac{\pi \tan ^{2} \beta}{H^{2}} x^{2}\right],
$$

where $S_{\omega}$ is the area of the ellipse section after convergence, obtained by $S_{\omega}=\pi(R-3 / 4 G) \times(R-1 / 4 G)$.

In the $\mathrm{S}-3$ mode, the rigid settlement of tunnel section is G/4. According to the superposition principle, the total ground surface settlement due to the convergence from region $\Omega$ to region $\omega$ by tunnel excavation can be expressed as follows:

$$
W(x)=W_{\Omega}(x)-W_{\omega}(x)=\frac{\left(\pi G R-3 / 16 \pi G^{2}\right) \tan \beta}{(H+G / 4)} \exp \left[-\frac{\pi \tan ^{2} \beta}{(H+G / 4)^{2}} x^{2}\right] .
$$

Correspondingly, the horizontal displacement is expressed as

$U(x)=\frac{\left(\pi G R-3 / 16 \pi G^{2}\right) \tan \beta \times x}{(H+G / 4)} \exp \left[-\frac{\pi \tan ^{2} \beta}{(H+G / 4)^{2}} x^{2}\right]$.
4.2. Simplified Formula for Twin Tunnel. The ground surface settlement and the horizontal displacement caused by the left tunnel, as illustrated in Figure 3(b), are expressed as

$$
\begin{aligned}
& W_{I}(x)=\frac{\left(\pi G R-3 / 16 \pi G^{2}\right) \tan \beta}{(H+G / 4)} \exp \left[-\frac{\pi \tan ^{2} \beta}{(H+G / 4)^{2}}\left(x+\frac{L}{2}\right)^{2}\right], \\
& U_{I}(x)=\frac{\left(\pi G R-3 / 16 \pi G^{2}\right) \tan \beta \times(x+L / 2)}{(H+G / 4)^{2}} \exp \left[-\frac{\pi \tan ^{2} \beta}{(H+G / 4)^{2}}(x+L / 2)^{2}\right] .
\end{aligned}
$$

Similarly, the ground surface settlement and the horizontal displacement of the right tunnel are written as

$$
\begin{aligned}
& W_{I I}(x)=\frac{\left(\pi G R-3 / 16 \pi G^{2}\right) \tan \beta}{(H+G / 4)} \exp \left[-\frac{\pi \tan ^{2} \beta}{(H+G / 4)^{2}}\left(x-\frac{L}{2}\right)^{2}\right], \\
& U_{I I}(x)=\frac{\left(\pi G R-3 / 16 \pi G^{2}\right) \tan \beta \times(x-L / 2)}{(H+G / 4)^{2}} \exp \left[-\frac{\pi \tan ^{2} \beta}{(H+G / 4)^{2}}\left(x-\frac{L}{2}\right)^{2}\right] .
\end{aligned}
$$

According to the superposition theory, the settlement and horizontal displacement of the ground surface in case of twin tunnel can be written as

$$
\begin{gathered}
W(x)=W_{I}(x)+W_{I I}(x), \\
U(x)=U_{I}(x)+U_{I I}(x) .
\end{gathered}
$$

4.3. Determination of the Gap Parameter G. Supposing that the ground loss ratio is $V_{l}$, which is defined as the percentage of the surface subsidence volume to that of the tunnel excavation in unit length, the relationship between $V_{l}$ and the gap parameter $G$ could be expressed as follows [6]: 


$$
V_{l}=\frac{\pi(R+G / 2)^{2}-\pi R^{2}}{\pi R^{2}}=\frac{G^{2}+4 G R}{4 R^{2}} .
$$

Hence, the gap parameter $G$ can be obtained from equation (13) as follows:

$$
G=2 R\left(\sqrt{1+V_{l}}-1\right) .
$$

According to Peck's study [4], the parameter of the ground loss ratio $V_{l}$ can be obtained by the maximum ground surface settlement $S_{\max }$ above the center of the tunnel. The relationship between the maximum ground settlement and ground loss is as follows:

$$
S_{\max }=\frac{\sqrt{\pi} R^{2} V_{l}}{\sqrt{2} i},
$$

where $i$ is the width of the surface settlement trough. Based on equation (12), the expression of $V_{l}$ and $S_{\max }$ can be obtained as

$$
V_{l}=\frac{\sqrt{2} i S_{\max }}{\sqrt{\pi} R^{2}} .
$$

For the width of the surface settlement trough, Peck also suggested another formula as shown in equation (17) using the measured data including the radius of tunnel $R$, the ground cover $H$, and the soil conditions.

$$
i=R\left[\frac{H}{(2 R)}\right]^{n}, \quad n=0.8 \sim 1 .
$$

Softer groundmass will lead to a greater value of $n$. When there are multiple ground layers above the tunnel opening, it is suggested to use the weight mean method to calculate the parameter $n$. Incorporating equation (13) into equation (16), the ground loss ratio could be obtained as follows:

$$
V_{l}=\frac{\sqrt{2}[H /(2 R)]^{n} S_{\max }}{\sqrt{\pi} R}, \quad n=0.8 \sim 1 .
$$

Substituting equation (18) into equation (14), the gap parameter $G$ can be expressed as follows:

$$
G=2 R \sqrt{1+\frac{\sqrt{2}[H /(2 R)]^{n} S_{\max }}{\sqrt{\pi} R}}-1, \quad n=0.8 \sim 1 .
$$

4.4. Determination of the Influence Angle $\beta$. For Knothe [34], the main influence range is expressed with $r(H)$, and $\beta$ is also called the main influence angle on the surface, as illustrated in Figure 4. Their relationship could be expressed as follows:

$$
r(H)=\frac{H}{\tan \beta},
$$

where $H$ is the depth of excavation. Another formula after comparing their study with Peck formula and stochastic medium theory is suggested as follows [26]:

$$
r(H)=\sqrt{2 \pi} \times i,
$$

where $i$ is the distance from the tunnel center. Substituting equation (21) into equation (20), $\tan \beta$ could be expressed as shown in

$$
\tan \beta=\frac{H}{\sqrt{2 \pi} \times i}=\frac{H}{2.5 i}
$$

Substitute equation (17) into equation (22). The formula of $\tan \beta$ is obtained as follows:

$$
\tan \beta=\frac{H}{2.5 R[H /(2 R)]^{n}} .
$$

In case of an elliptical tunnel section, the average radius $R$ can be obtained using the major and minor axis aforementioned. With the gap parameter $G$ and the tangential value of the influence angle $\beta$ discussed above, the surface settlement and horizontal displacement could be obtained using the simplified procedure.

\section{Validation of the Simplified Solution}

To validate the accuracy of the simplified solution, the results predicted by the simplified procedure for five cases are compared with those obtained by the SM theory and measured in situ. The five tunnel cases are the Urumqi City Metro Line 1 Tunnel in China, Heathrow Express Trial Tunnel in London, Barcelona Subway Tunnel in Spain, LIU Yanghe Tunnel in China, and Pushan Subway $1{ }^{\text {st }}$ Line tunnel in South Korea. Table 1 lists the calculation parameters of the tunnel for five cases.

The former four tunnels in Table 1 are for a single tunnel case. The maximum ground settlement observed in situ is $60.8 \mathrm{~mm}, 40 \mathrm{~mm}, 25 \mathrm{~mm}$, and $37 \mathrm{~mm}$ for the Urumqi City Metro Line 1 Tunnel, Heathrow Express Trial Tunnel, Barcelona Subway Tunnel, LIU Yanghe Tunnel. Using equations (16) and (20) according to the measured data, the corresponding gap parameter $G$ could be obtained as $38 \mathrm{~mm}$, $58 \mathrm{~mm}, 31 \mathrm{~mm}, 81 \mathrm{~mm}$, and $29 \mathrm{~mm}$. Using equation (20) according to the measured data, the value of $\tan \beta$ is calculated to be $0.75,0.929,0.82,0.84$, and 0.8 . The predicted maximum ground settlements by the simplified procedure are $60.59 \mathrm{~mm}, 38.14 \mathrm{~mm}, 31.87 \mathrm{~mm}$, and $37.20 \mathrm{~mm}$ for the four tunnels using equation (7). The predicted maximum ground settlements predicted by the SM theory are $55.03 \mathrm{~mm}, 39.29 \mathrm{~mm}, 33.27 \mathrm{~mm}$, and $40.73 \mathrm{~mm}$. The difference between the simplified solution and the measured data is $0.35 \%, 4.65 \%, 27.48 \%$, and $0.54 \%$. The difference between the results predicted by the SM theory and the measured data is $9.49 \%, 1.78 \%, 33.08 \%$, and $10.08 \%$. The ground settlement curve predicted by the SM theory and the simplified procedure is plotted in Figure 5 together with the measured data. It can be seen that the predicted ground settlement curve agrees well with those predicted by the SM theory and measured in situ even though the maximum ground settlements are slightly underpredicted by the simplified procedure.

Pushan Subway $1^{\text {st }}$ Line Tunnel is considered an example of a twin tunnel case. The tunnel is located in South Korea with a length of $5.3 \mathrm{~km}$. The NATM was adopted in its 




FIgURE 4: Influence zone of ground settlement and influence angle $\beta$.

TABLE 1: Calculation parameters of case studies [6, 29, 35-37].

\begin{tabular}{|c|c|c|c|c|c|}
\hline Tunnel name & $\begin{array}{l}\text { Urumqi City Metro } \\
\text { Line 1, Xinjiang }\end{array}$ & $\begin{array}{l}\text { Heathrow Express Trial } \\
\text { Tunnel, London }\end{array}$ & $\begin{array}{l}\text { Barcelona Subway } \\
\text { Tunnel, Spain }\end{array}$ & $\begin{array}{c}\text { Liu Yanghe Tunnel } \\
\text { DK1569+300, Changsha }\end{array}$ & $\begin{array}{l}\text { Pushan Subway } \\
1^{\text {st }} \text { Line, South } \\
\text { Korea }\end{array}$ \\
\hline Strata condition & $\begin{array}{l}\text { Soft soil with } \\
\text { thickness of } 5-15 \mathrm{~m}\end{array}$ & $\begin{array}{l}0-2 \mathrm{~m} \text { fill ground, } 2-4 \mathrm{~m} \\
\text { terrace gravel, }>4 \mathrm{~m} \text { stiff } \\
\text { London clay }\end{array}$ & $\begin{array}{l}\text { Red and brown clay } \\
\text { with some gravel }\end{array}$ & $\begin{array}{l}\text { Grade V surrounding } \\
\text { strata with gravel }\end{array}$ & $\begin{array}{l}\text { Sandy clay with } \\
\text { gravel and silty } \\
\text { sand }\end{array}$ \\
\hline $\begin{array}{l}\text { Tunnel depth } H \\
\text { (m) }\end{array}$ & 8.1 & 19 & 10 & 20 & 16 \\
\hline Main axis $A(\mathrm{~m})$ & 6.39 & 4.25 & 4 & 4 & 5 \\
\hline $\begin{array}{l}\text { Minor axis } B \\
(\mathrm{~m})\end{array}$ & 4.6 & 4.25 & 4 & 3 & 3 \\
\hline$S_{\max }(\mathrm{mm})$ & 60.8 & 40 & 25 & 37 & 6.20 \\
\hline$G(\mathrm{~mm})$ & 38 & 58 & 31 & 81 & 29 \\
\hline $\tan \beta$ & 0.75 & 0.929 & 0.82 & 0.84 & 0.8 \\
\hline $\begin{array}{l}\text { Construction } \\
\text { method }\end{array}$ & $\begin{array}{l}\text { Undercutting } \\
\text { method }\end{array}$ & Open Shield & Shield & $\begin{array}{l}\text { Benching Tunneling } \\
\text { Method }\end{array}$ & NATM \\
\hline
\end{tabular}



SM theory

- - Simplified solution

- Measured data

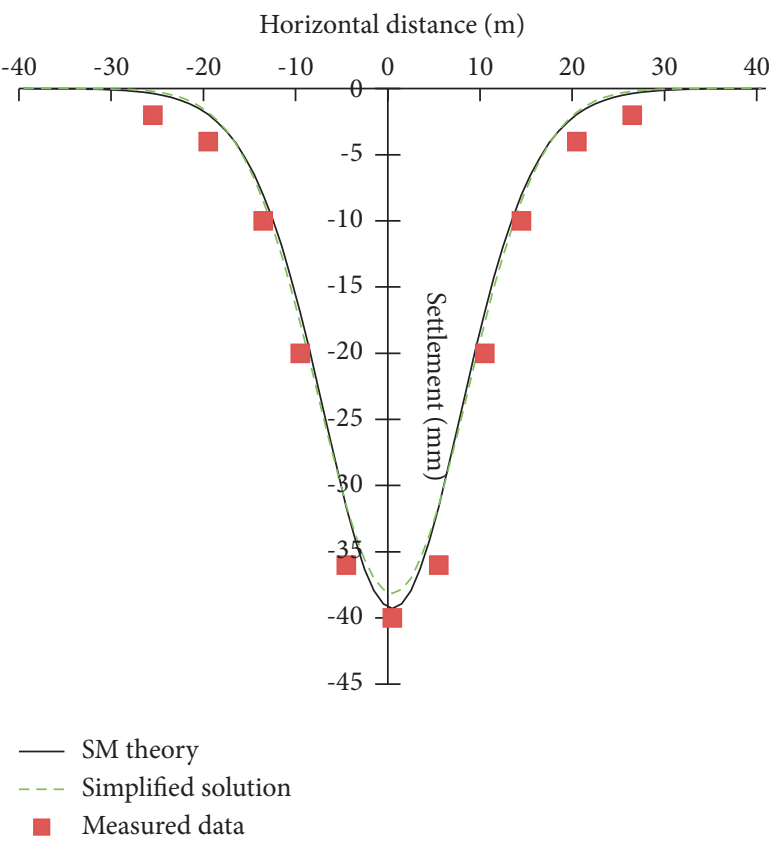

(a)

(b)

Figure 5: Continued. 




(c)

(d)

Figure 5: Measured and analytical ground surface settlement. (a) Urumqi City Metro Line 1 Tunnel in China; (b) Heathrow Express Trial Tunnel in London; (c) Barcelona Subway Tunnel in Spain; (d) Liu Yanghe Tunnel in China.

construction, and excavation was carried out in two parts: upper and lower parts [35]. According to the monitored section, the twin circular tunnels have a diameter $R$ of $10.0 \mathrm{~m}$ and depth $H$ of $16.0 \mathrm{~m}$. This section was driven through the ground composed of weathered rock, sand with gravel, and sandy clay [35]. The observed maximum ground settlement is $6.20 \mathrm{~mm}$. Using equation (16) according to the measured data, the corresponding gap parameter $G$ could be obtained to be $29 \mathrm{~mm}$. Using equation (20) according to the measured data, the value of $\tan \beta$ is calculated to be 0.80 . The ground horizontal displacement for the twin tunnels is predicted by the simplified procedure using equation (12). Due to measured data limitations in pieces of literature, the ground displacements of the right tunnel are used in the current study. The ground horizontal displacement predicted by the SM theory and the simplified procedure is plotted in Figure 6 together with the measured data. The predicted ground surface horizontal displacement curve predicted by the simplified procedure agrees well with those predicted by the SM theory and measured in situ.

The comparison for the five tunnels illustrates that the simplified formula can provide a more reasonable prediction for the ground movement induced by tunneling when the gap parameter $G$ and influence angle $\beta$ are available. The tunneling-induced ground movement for the single tunnel with a circular cross section, which is the special case, could be predicted accurately and reasonably by the simplified procedure. For tunnels with noncircular cross sections, the simplified procedure still has enough accuracy to predict tunneling-induced ground movement. Besides, for the twintunnel case as shown in Figure 6, the tunneling-induced ground movement could also be predicted with a high accuracy compared to those obtained by the SM theory and

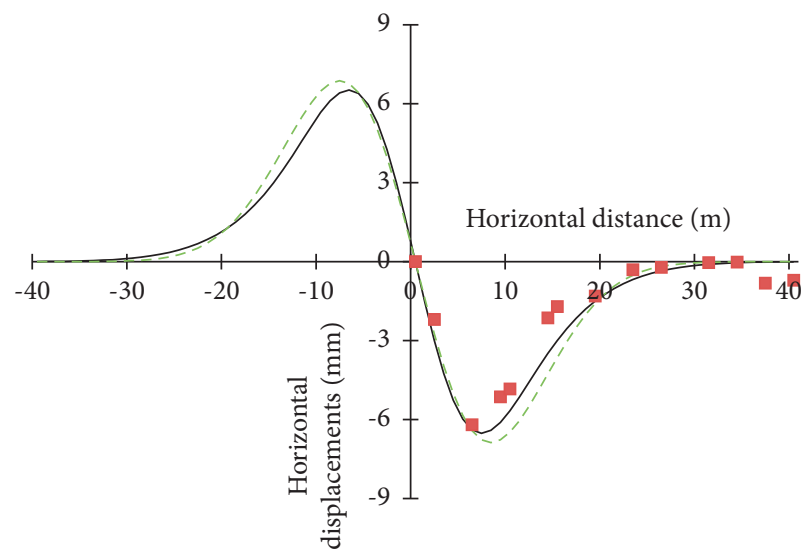

_ SM theory
- - Simplified solution

FIGURE 6: Measured and analytical ground horizontal displacement of Pushan Subway $1^{\text {st }}$ Line Tunnel.

measured in situ. A feasible approach is developed to estimate these two parameters using the maximum ground settlement $S_{\max }$ and tunnel design parameters, including tunnel depth $H$ and diameter $R$.

\section{Conclusions}

Comparing to the general approaches based on the stochastic medium theory, this study takes a simplified way to predict the tunneling-induced ground movement. The condition for this simplified approach application is the 
same as that for the SM method, which is for the shallow-buried tunnel condition. With the simplified formulas, the ground movement for single tunnel and twin tunnels could be predicted based on the gap parameter $G$ and influence angle $\beta$. A feasible approach is developed to estimate these two parameters using the maximum ground settlement Smax and tunnel design parameters, including tunnel depth $H$ and diameter $R$.

To validate its accuracy, the ground movement predicted by the simplified procedure is compared with that obtained by the SM theory and measured in situ for five tunnel cases, including the Urumqi City Metro Line 1 Tunnel in China, Heathrow Express Trial Tunnel in London, Barcelona Subway Tunnel in Spain, LIU Yanghe Tunnel in China, and Pushan Subway $1^{\text {st }}$ Line tunnel in South Korea. It can be observed that the predicted tunneling-induced-ground movement for single and twin-tunnel cases with circular and noncircular cross sections agree well with those obtained by the SM theory and measured in situ. It can be concluded that the simplified formula can reduce the calculation work and provide a more reasonable prediction for the ground movement induced by tunneling.

\section{Appendix}

\section{A. The Boundary Parameters for Single Tunnel Model}

Considering the nonuniform boundary condition, the boundary parameters $(a, b, c, d)$ of the initial tunnel cross section $\Omega$ for a single tunnel are expressed as

$$
\begin{aligned}
& a=-A, \\
& b=A, \\
& c=H-B \sqrt{1-\left(\frac{x}{A}\right)^{2},} \\
& d=H+B \sqrt{1-\left(\frac{x}{A}\right)^{2}},
\end{aligned}
$$

where $x$ is the distance from the center of the $x$ axis, as shown in Figure 3.

Furthermore, the boundary parameters $(e, f, g, h)$ of the tunnel cross section $\omega$ after convergence for single tunnel could be expressed as

$$
\begin{aligned}
& e=-\left(A-u_{0}+u_{\theta}\right), \\
& f=A-u_{0}+u_{\theta}, \\
& g=H+u_{z}-\left(B-u_{0}+u_{\theta}\right) \sqrt{1-\left(\frac{x}{A-u_{0}+u_{\theta}}\right)^{2}}, \\
& h=H+u_{z}+\left(B-u_{0}-u_{\theta}\right) \sqrt{1-\left(\frac{x}{A-u_{0}+u_{\theta}}\right)^{2}} .
\end{aligned}
$$

\section{B. The Boundary Parameters for the Twin- Tunnel Model}

As the analysis process of the single tunnel, the boundary parameters of the left tunnel could be expressed as

$$
\begin{aligned}
& a_{1}=-A-\frac{L}{2}, \\
& b_{1}=A-\frac{L}{2}, \\
& c_{1}=H-B \sqrt{1-\left(\frac{x+L / 2}{A}\right)^{2},} \\
& d_{1}=H+B \sqrt{1-\left(\frac{x+L / 2}{A}\right)^{2},} \\
& e_{1}=-\left(A-u_{0}+u_{\theta}\right)-\frac{L}{2}, \\
& f_{1}=A-u_{0}+u_{\theta}-\frac{L}{2},
\end{aligned}
$$$$
g_{1}=H+u_{z}-\left(B-u_{0}+u_{\theta}\right) \sqrt{1-\left(\frac{x+L / 2}{A-u_{0}+u_{\theta}}\right)^{2}}
$$$$
a_{11}=-A+\frac{L}{2}
$$$$
b_{11}=A+\frac{L}{2}
$$

$$
c_{11}=H-B \sqrt{1-\left(\frac{x-L / 2}{A}\right)^{2}} \text {, }
$$$$
d_{11}=H+B \sqrt{1-\left(\frac{x-L / 2}{A}\right)^{2}}
$$$$
e_{11}=-\left(A-u_{0}+u_{\theta}\right)-\frac{L}{2}
$$$$
f_{11}=A-u_{0}+u_{\theta}+\frac{L}{2}
$$

$g_{11}=H+u_{z}-\left(B-u_{0}+u_{\theta}\right) \sqrt{1-\left(\frac{x-L / 2}{A-u_{0}+u_{\theta}}\right)^{2}}$

$$
h_{11}=H+u_{z}-\left(B-u_{0}-u_{\theta}\right) \sqrt{1-\left(\frac{x-L / 2}{A-u_{0}+u_{\theta}}\right)^{2}}
$$




\section{Data Availability}

The data used to support the findings of this study are included in the article.

\section{Conflicts of Interest}

The authors declare that there are no conflicts of interest.

\section{Acknowledgments}

This work was funded by the National Natural Science Foundation of China (no. 52109132), Shandong Provincial Natural Science Foundation (no. ZR2020QE270), Key Laboratory of Rock Mechanics and Geohazards of Zhejiang Province, China (no. ZJRMG-2020-03), Key Laboratory of Deep Earth Science and Engineering (Sichuan University), Ministry of Education, China (no. DESE202109), and Key Laboratory of Mining Disaster Prevention and Control (Shandong University of Science and Technology), Ministry of Education, China (no. JMDPC202109).

\section{References}

[1] D. Lin, J. D. Nelson, M. Beecroft, and J. Cui, “An overview of recent developments in China's metro systems," Tunnelling and Underground Space Technology, vol. 111, p. 103783, 2021.

[2] L. Ma, L. Ding, and H. Luo, "Non-linear description of ground settlement over twin tunnels in soil," Tunnelling and Underground Space Technology, vol. 42, pp. 144-151, 2014.

[3] S. R. Dindarloo and E. Siami-Irdemoosa, "Maximum surface settlement based classification of shallow tunnels in soft ground," Tunnelling and Underground Space Technology, vol. 49, pp. 320-327, 2015.

[4] R. B. Peck, "Deep Excavations and tunneling in soft ground," in Proceedings of the 7th International Conference on Soil Mechanics and Foundation Engineering, pp. 225-290, Mociedad Mexicana de Mecanica, Mexico City, April 1969.

[5] F. Wu, H. Jin, Y. Shang et al., "Study of building/structure settlement prediction method surrounding urban rail transit tunnel engineering," Chinese Journal of Rock Mechanics and Engineering, vol. 32, no. 2, pp. 3535-3544, 2013.

[6] N. Loganathan and H. G. Poulos, "Analytical prediction for tunneling-induced ground movements in clays," Journal of Geotechnical and Geoenvironmental Engineering, vol. 124, no. 9, pp. 846-856, 1998.

[7] X. L. Yang and J. M. Wang, "Ground movement prediction for tunnels using simplified procedure," Tunnelling and Underground Space Technology, vol. 26, no. 3, pp. 462-471, 2011.

[8] B. Li and Z. Z. Wang, "Numerical study on the response of ground movements to construction activities of a metro station using the pile-beam-arch method," Tunnelling and Underground Space Technology, vol. 88, pp. 209-220, 2019.

[9] Z. Zhong, C. Li, X. Liu, Y. Fan, and N. Liang, "Analysis of ground surface settlement induced by the construction of mechanized twin tunnels in soil-rock mass mixed ground," Tunnelling and Underground Space Technology, vol. 110, no. 1, p. 103746, 2021.

[10] A. Franza, B. Zhou, and A. M. Marshall, "The effects of relative tunnel depth and volume loss on vertical settlements above tunnels in dense sands," Geo-China Int. Conf.vol. 21, pp. 125-132, 2016.
[11] M. Hasanipanah, M. Noorian-Bidgoli, D. Jahed Armaghani, and H. Khamesi, "Feasibility of PSO-ANN model for predicting surface settlement caused by tunneling," Engineering with Computers, vol. 32, no. 4, pp. 705-715, 2016.

[12] N. Zhang, A. Zhou, Y. Pan, and S.-L. Shen, "Measurement and prediction of tunnelling-induced ground settlement in karst region by using expanding deep learning method," Measurement, vol. 183, Article ID 109700, 2021.

[13] N. Zhang, S.-L. Shen, A. Zhou, and Y.-F. Jin, "Application of LSTM approach for modelling stress-strain behaviour of soil," Applied Soft Computing, vol. 100, p. 106959, 2021.

[14] F. Wang, B. Gou, and Y. Qin, "Modeling tunneling-induced ground surface settlement development using a wavelet smooth relevance vector machine," Computers and Geotechnics, vol. 54, pp. 125-132, 2013.

[15] L. Zhang, X. Wu, W. Ji, and S. M. AbouRizk, "Intelligent approach to estimation of tunnel-induced ground settlement using wavelet packet and support vector machines," Journal of Computing in Civil Engineering, vol. 31, no. 2, Article ID 4016053, 2017.

[16] S. L. Song, S. L. Shen, N. Zhang et al., "Modelling the performance of EPB shield tunnelling using machine and deep learning algorithms," Geoscience Frontiers, vol. 12, no. 5, Article ID 101177, 2021.

[17] W. Zhang, C. Wu, H. Zhong, Y. Li, and L. Wang, "Prediction of undrained shear strength using extreme gradient boosting and random forest based on Bayesian optimization," Geoscience Frontiers, vol. 12, no. 1, pp. 469-477, 2021.

[18] W. Zhang, J. Ching, A. T. C. Goh, and A. Y. F. Leung, "Big data and machine learning in geoscience and geoengineering: introduction," Geoscience Frontiers, vol. 12, no. 1, pp. 327-329, 2021.

[19] A. Verruijt and J. R. Booker, "Surface settlements due to deformation of a tunnel in an elastic half plane," Géotechnique, vol. 46, no. 4, pp. 753-756, 1996.

[20] J. G. Agar, N. R. Morgenstern, and J. D. Scott, "Shear strength and stress-strain behaviour of Athabasca oil sand at elevated temperatures and pressures," Canadian Geotechnical Journal, vol. 24, no. 1, pp. 1-10, 1987.

[21] R. Baker, "Nonlinear mohr envelopes based on triaxial data," Journal of Geotechnical and Geoenvironmental Engineering, vol. 130, no. 5, pp. 498-506, 2004.

[22] M. Cai, H. Morioka, P. K. Kaiser et al., "Back-analysis of rock mass strength parameters using AE monitoring data," International Journal of Rock Mechanics and Mining Sciences, vol. 44, no. 4, pp. 538-549, 2007.

[23] M. Cai, P. K. Kaiser, Y. Tasaka, and M. Minami, "Determination of residual strength parameters of jointed rock masses using the GSI system," International Journal of Rock Mechanics and Mining Sciences, vol. 44, no. 2, pp. 247-265, 2007.

[24] J. Litwiniszyn, "Fundamental principles of the mechanics of stochastic medium," in Proceedings of the 3rd Conference Theoretical Applying Mechanics, pp. 93-110, Springer, Bongalore, India, 1957.

[25] B. Liu, W. Yang, G. Zhang et al., "A prediction model based on stochastic medium theory for ground surface settlement induced by non-uniform tunnel deformation," Chinese Journal of Rock Mechanics and Engineering, vol. 37, no. 8, pp. 1943-1952, 2018.

[26] X. Han and N. Li, "Comparative analysis of strata prediction models for ground movement induced by tunnel construction," Chinese Journal of Rock Mechanics and Engineering, vol. 26, pp. 594-600, 2007.

[27] Z. Z. Wu, "Stochastic medium predicting model of ground movement tunneling based on non-uniform convergence 
mode," J. Cent. South Uni.(Sci. Tech.), vol. 41, pp. 2005-2010, 2010.

[28] C. González and C. Sagaseta, "Patterns of soil deformations around tunnels. Application to the extension of Madrid Metro," Computers and Geotechnics, vol. 28, no. 6-7, pp. 445-468, 2001.

[29] C. Wang, Y. J. Qin, Y. U. Guang-Ming et al., "Study on prediction of ground surface settlement caused by construction of shallow buried tunnel based on non-uniform random medium model," Railway Stand. Design, pp. 104-109, 2018.

[30] K. M. Lee, R. K. Rowe, and K. Y. Lo, "Subsidence owing to tunnelling. I. Estimating the gap parameter," Canadian Geotechnical Journal, vol. 29, no. 6, pp. 929-940, 1992.

[31] B. C. Liu, "Ground surface movements due to underground excavation in the PR China," in Comprehensive Rock Engineering, J. A. Hudson, Ed., , pp. 781-817, Pergamon Press, 1993.

[32] L. Tong, K. H. Xie, Y. F. Cheng et al., "Elastic solution of sallow tunnels in clays considering oval deformation of ground," Rock and Soil Mechanics, vol. 30, pp. 393-398, 2009.

[33] K.-H. Park, "Analytical solution for tunnelling-induced ground movement in clays," Tunnelling and Underground Space Technology, vol. 20, no. 3, pp. 249-261, 2005.

[34] S. Knothe, "Observations of surface movements under influence of mining and their theoretical interpretation," in Proceedings of the European Conference on Ground Movement, pp. 210-218, University of Leeds, Leeds, UK, 1957.

[35] S. W. Hong and G. J. Bae, "Ground movements associated with subway tunneling in Korea," in Proceedings of the Underground Construction in Soft Ground, pp. 229-232, Balkema, Rotterdam, 1995.

[36] J. S. Yang, B. C. Liu, and M. C. Wang, "Modeling of tunnelinginduced ground surface movements using stochastic medium theory," Tunnelling and Underground Space Technology, vol. 19, pp. 112-123, 2004.

[37] W. Luo, Stability Analysis and Reliability Research on the Shallow Tunnel under Unsymmetrical Pressure, Ph.D. Thesis, Central South University, Changsha, 2014. 